\title{
Investigation and Research on Safety Related Individual Factors of Mountainous Expressway Professional Driver
}

\author{
LinFu Zhou
}

Sichuan Vocational and Technical College of Communications, P.O. Box: Wenjiang District, Liu Tai Road, No. 208,Chengdu, Sichuan, 611130

scjyzlf@126.com

Keywords: mountainous expressway; professional driver; safety; individual factors.

Abstract. A sampling survey was conducted on Professional Driver of mountainous expressway in Sichuan Province. The statistical software SPSS 13.0 was used to analyze the survey data in order to know living condition, safety consciousness and driving behavior of Professional Driver of mountainous expressway and to lay a foundation of further study on security assurance.

\section{Introduction}

At present, the Chinese mountainous expressway transit accident rate extremely is high, has created the massive loss to the society, and simultaneously has also brought the disaster to the family. Creates this matter the reason, is our country transport business present situations and so on drivers' social position, current economic condition. Poor social living conditions of the driver during transport are often in overloading, fatigue driving status, and planted a hidden danger for traffic accidents.

Therefore, in this article to Sichuan mountain expressway drivers for the study, conducted an in-depth survey and analysis .Grasps the pilot from the basis to survive present situations and so on condition, driving behavior, safe consciousness, regarding this and carries on the research analysis , and puts forward the safety control proposal to the mountainous expressway transport business drivers driving. This article to enhances our country mountainous area highway operation security present situation to have the vital practical significance.

\section{The mountainous expressway safety association individual professional driver sample to form}

In order to guarantee the investigation the scientific nature, the research uses hives off the random sampling, passes through the mountainous expressway the area the professional driver to divide into the freight vehicle professional driver, the passenger train professional driver, the tourist car professional driver three categories. The actual 1787 questionnaires, of which the freight vehicle professional driver questionnaire (including dangerous goods professional drivers) of 676, the passenger train professional driver questionnaire (including the long term transport drivers) of 606, the tourist car professional driver questionnaire of 511 (figure 1).

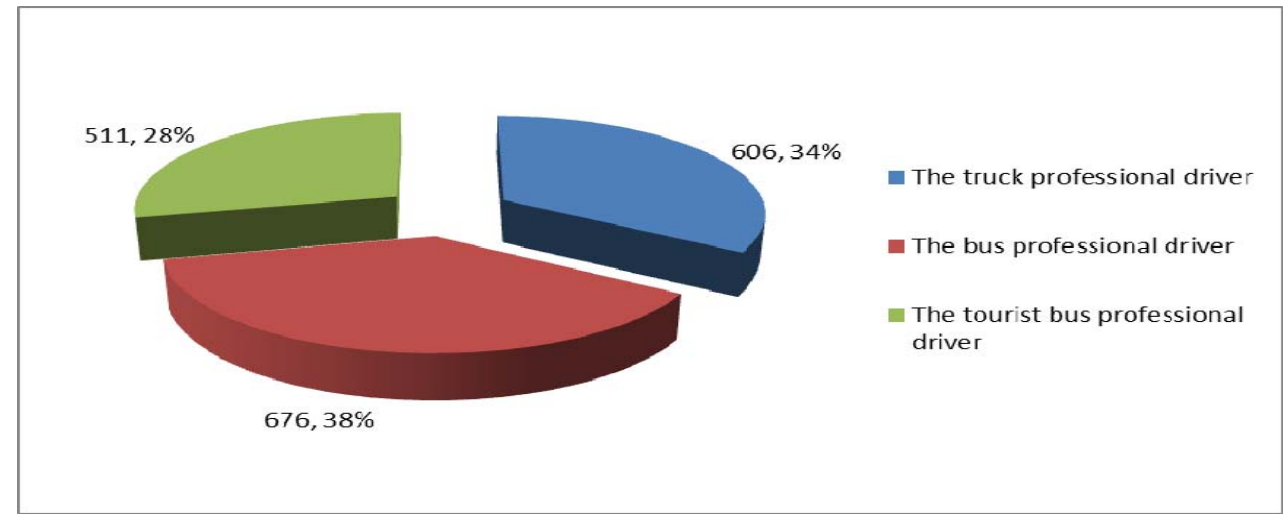

figure 1 The survey sample constituted diagram 


\section{The mountainous expressway professional driver basic condition and living environment analysis}

\section{Age and driving year's statistical analysis}

In 1691 drivers in statistics, maximum age 60 years, minimum age of 20 years, average age of 37.58 years. The structure driver's age on the whole reasonable, between the physical age of energetic, quick thinking, which fundamentally guaranteed driving safety. But the team decline in physiological function of middle-aged senior citizens, young person causes such as lack of experience, easily induced by traffic accidents.

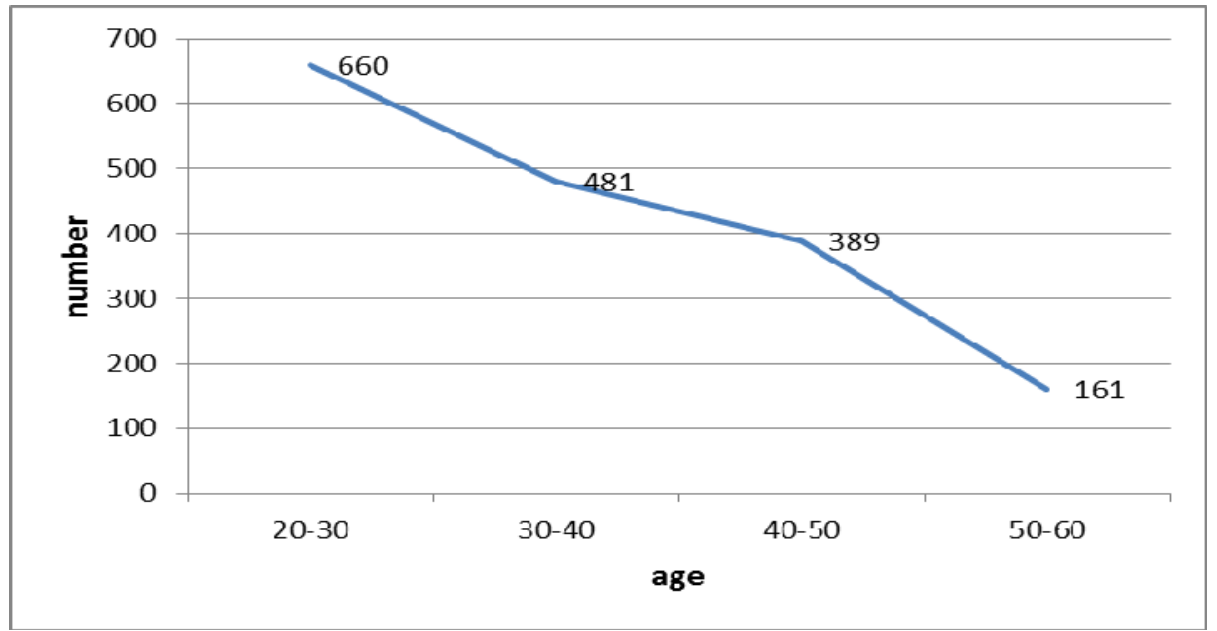

figure 2 Statistical analysis of professional driver's age structure of mountainous expressway

As shown in figure 2 shows, in the 1547 survey to be a driver, driving up for 45 years, the shortest for 2 years, average driving is 13.57 years. There is a 86 per cent of the professional driver's driving years is between 10 to 20 , between the driver has accumulated a certain amount of driving experience, it is a favorable condition for safe driving.

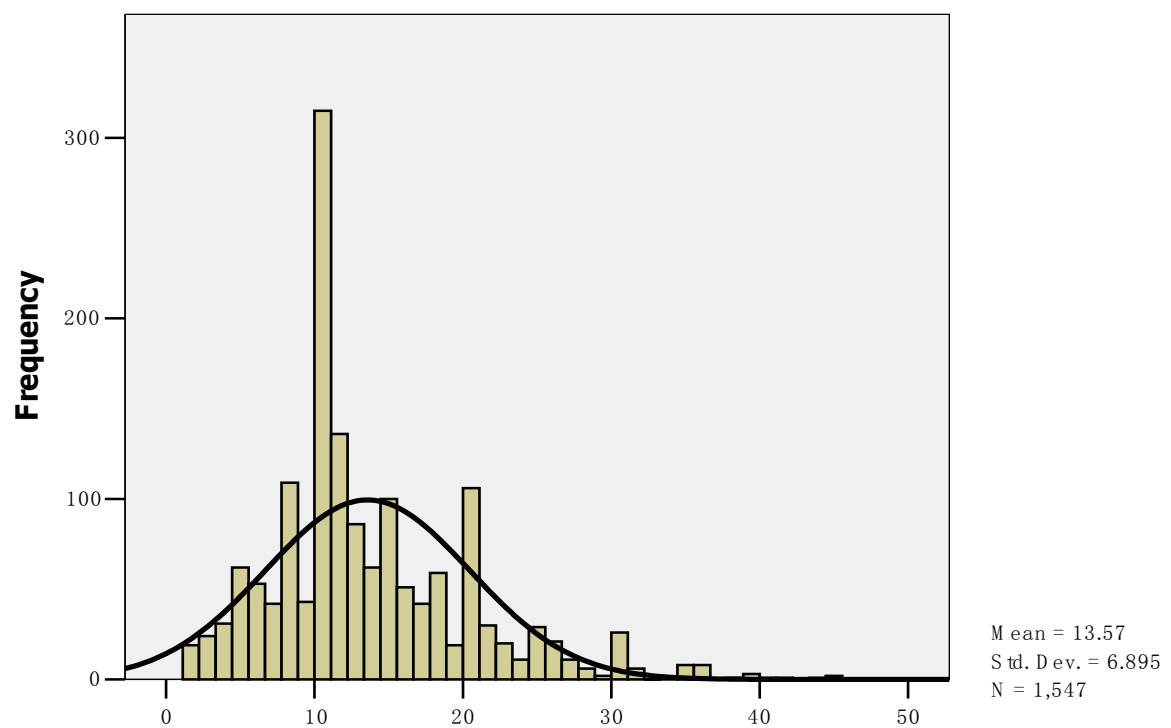

figure 3 Frequency chart of professional driver's driving years structure of mountainous expressway

\section{The statistical analysis of the driver and the composition of their family}

Figure 4 shows the driver's background. Cadre family background only 93 people, account for the total sample proportion are only 5.2\% .Most of the professional drivers was born lower-middle class family, concentrate on the workers and peasants family background, the ratio is about $89.7 \%$.It follows professional driver is a relatively disadvantaged group. 


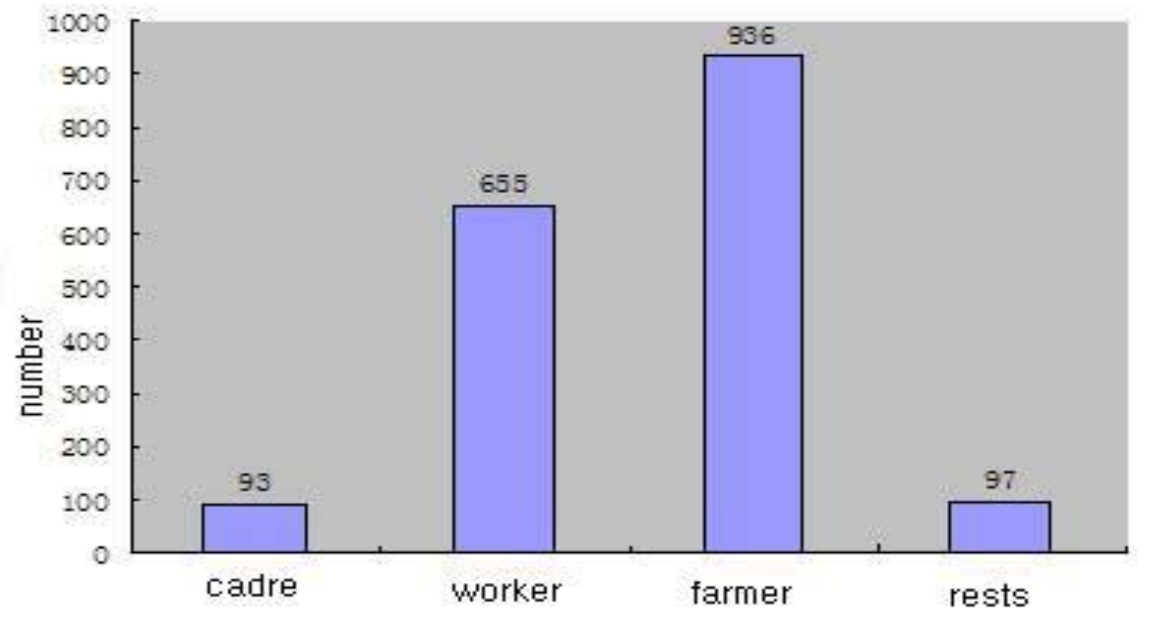

Figure4 Analysis of professional driver's family background structure of mountainous expressway

\section{Statistical analysis of the economic income of drivers and their families}

At present, professional driver facing greater pressure of life, when income survey results cannot relieve this pressure. Table 1 shows a sample of professional drivers of personal monthly income .In 1753 a valid data, $40 \%$ drivers' income is in 1200 Yuan of the following, $50 \%$ drivers' income is between 1200 Yuan and 2000 Yuan; only 10\% of drivers' income is higher than 2000 Yuan. It can be concluded that income of most professional drivers in mountainous expressway driving is low (In view of the actual income of the professional drivers and drivers have concerns and other reasons; the data may exist to a certain degree of distortion).

Table1 Personal income of mountain expressway professional drivers

\begin{tabular}{ccc}
\hline salary & quantity & ratio \\
\hline Less than 1000 & 699 & $39.9 \%$ \\
Yuan & & \\
$1000-2000$ & 881 & $50.3 \%$ \\
2000-3000 & 132 & $7.5 \%$ \\
More than 3000 & 41 & $2.3 \%$ \\
$\quad$ Yuan & & \\
total & 1753 & $100.0 \%$ \\
\hline
\end{tabular}

\section{Analysis of mountainous expressway professional driver safety awareness and safety knowledge}

As shown in Figure 5 is sampling of professional drivers ' education. In 1755 effective data, only $4.8 \%$ professional driver's education is the technical college and above, high school educated is occupied $37 \%$, junior middle school educated the is occupied $52 \%$, elementary school and following culture account for $7 \%$. We can see that the mountainous expressway professional drivers do not have a high level of education. Fair enough, they lack of knowledge about expressway traffic safety, vehicle technology and safe driving knowledge.

In order to realize the level of understanding of drivers for mountainous expressway long and large ramp, this study was devoted to setting related topics(See Table 3.1 ). Most drivers have a certain concepts for long and large ramp.92.42\% of drivers had to drive traffic through the long and large ramp experience, but their understanding for long and large ramp is not comprehensive. In investigation, there is $74.6 \%$ driver for long and large ramp without a more comprehensive understanding; there is $17.82 \%$ driver considered safe to deal with long and large ramp; there is $17.82 \%$ driver fully make nothing of the long and large ramp.

The investigation also found that nearly $20 \%$ of drivers for more than one year had not accepted security knowledge education (Table 2 ) 


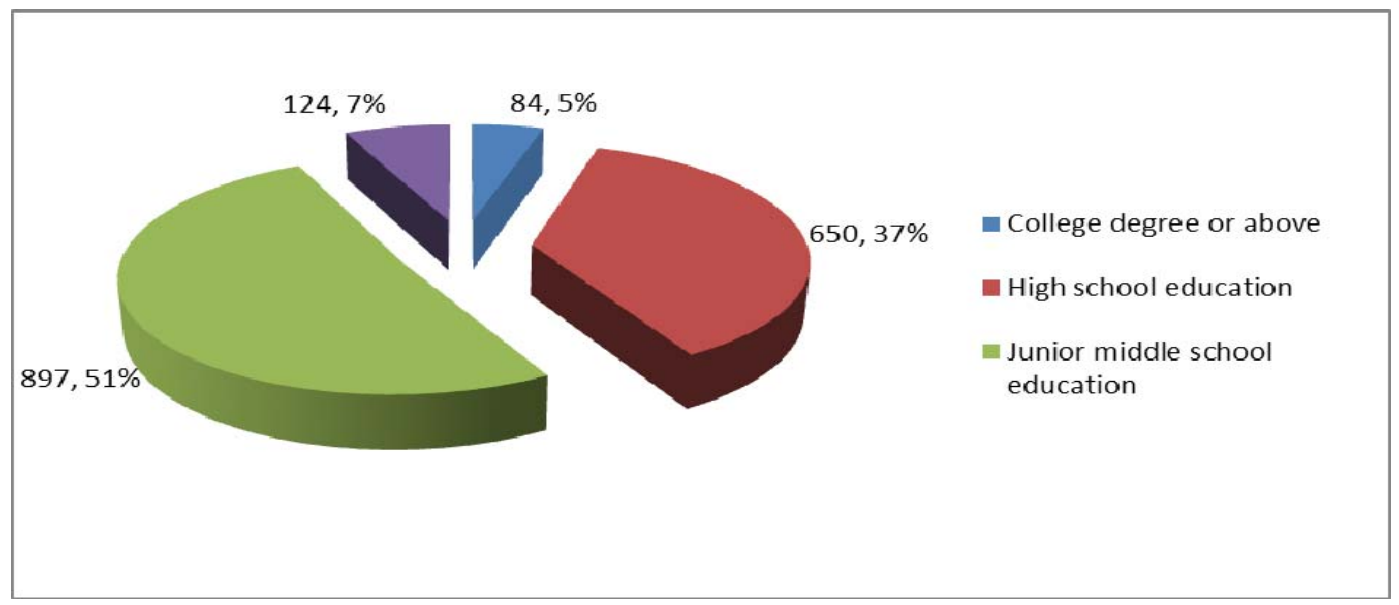

figure 2 The statistics mountain expressway professional driver education

Table2 The level of understanding of drivers for mountainous expressway long and large ramp

\begin{tabular}{ccc}
\hline understanding of long and large & quantity & ratio \\
ramp & & \\
\hline no concept & 128 & $7.58 \%$ \\
concept ,not comprehensive & 1,260 & $74.60 \%$ \\
concept ,Security response & 301 & $17.82 \%$ \\
total & 1,689 & $100.00 \%$ \\
\hline
\end{tabular}

Table3 Frequency analysis of safety education cycle of professional driver' of mountainous

\begin{tabular}{ccc}
\multicolumn{3}{c}{ expressway } \\
\hline $\begin{array}{c}\text { safety education } \\
\text { cycle }\end{array}$ & quantity & ratio \\
\hline 3 months & 546 & $37.6 \%$ \\
6 months & 379 & $26.1 \%$ \\
1 year & 253 & $17.4 \%$ \\
over 1 year & 273 & $18.8 \%$ \\
total & 1,451 & $100.0 \%$ \\
\hline
\end{tabular}

\section{Analysis of driving behavior of the mountainous expressway professional driver}

The research of expressway operation vehicle speed (Table 4 ) showed that there are only $1.2 \%$ of the vehicles had no speeding; there are $98.8 \%$ of the vehicles had speeding and there are $10.4 \%$ of vehicles had surpass $120 \mathrm{~km} / \mathrm{h}$ the driving behavior when vehicles travel on bidirectional four traffic lane mountainous expressway (speed limit $80 \mathrm{~km} / \mathrm{h}$, partial road section speed limit $60 \mathrm{~km} / \mathrm{h}$ ). It is great security implications of operating buses and goods vehicles.

Table 4 Highway vehicle speed behavior in Mountainous area

\begin{tabular}{ccc}
\hline $\begin{array}{c}\text { highway vehicle } \\
\text { speed }\end{array}$ & quantity & ratio \\
\hline $60-80 \mathrm{~km} / \mathrm{h}$ & 21 & $1.2 \%$ \\
$80-100 \mathrm{~km} / \mathrm{h}$ & 934 & $52.8 \%$ \\
$100-120 \mathrm{~km} / \mathrm{h}$ & 631 & $35.6 \%$ \\
over $120 \mathrm{~km} / \mathrm{h}$ & 184 & $10.4 \%$ \\
total & 1,770 & $100.0 \%$ \\
\hline
\end{tabular}

One of the main causes of the accident is fatigue driving. The investigation indicated more than $11 \%$ of the driver's continuous driving time of more than seven hours. Even there is individual operator's continuous driving time even more than 12 hours, as shown in table 4 . 


\section{Summary}

According to the survey and analysis, the driver working in the mountainous expressway has some difficultis, such as high life pressure, low social status, these problems will lead to their unhealthy psychological and reduce the sense of responsibility. In order to pursue the economic interest, the driver's behavior of break traffic regulations will increase. This behavior lays down the hidden danger for the mountainous expressway safe transportation. The fundamental cause of mountain expressway accidents is driver's safety awareness is weak, and lack of knowledge about mountain expressway characteristics, lack of knowledge and technical capacity for the safe transport of lack of mountain expressway.

Table 5 Analysis mountain expressway operation driver's continuous driving time

\begin{tabular}{ccc}
\hline continuous driving time & quantity & ratio \\
\hline 1-2 hours & 464 & $27.3 \%$ \\
3-4 hours & 747 & $44.0 \%$ \\
5-6 hours & 301 & $17.7 \%$ \\
7 hours & 187 & $11.0 \%$ \\
total & 1,699 & $100.0 \%$ \\
\hline
\end{tabular}

Road traffic system consisting of three elements is people, vehicles and road environments. If three elements not harmony, it could lead to traffic accidents. Usually, when road traffic accident cause factor investigation, the driver's improper operation is the main cause of the accident, is also the most direct cause of traffic accidents. Because the mountainous expressway be limited by the terrain environment, road linear relatively poor, such as the curve, the bridge, the tunnel, the long and large ramp and so on the special road section is multitudinous, the road design parameter has used some limit standard, therefore the driver in driving the process of negligence or improper driving behavior may lead to accidents. In order to effectively control the occurrence of traffic accidents on mountainous expressways, based on the professional driver's investigation present situation, this article puts forward following several traffic safety safeguard proposal.

(1)Improve driver's safety awareness and legal awareness

As a scientific summary of the years of experience in road safety, traffic laws to ensure traffic safety plays a very important role. In order to ensure road safety, drivers must raise legal awareness, and to strictly observe traffic regulations. Because the traffic safety education is an important means to enhance safety awareness and legal awareness, therefore driver's traffic safety education must be strengthened. We need to improve traffic safety to the height of the traffic safety culture to understand, so that every driver has a strong safety requirements, and training driver "I want security" habits and conscious action.

(2)Have technology for mountainous expressway safe driving

In addition to mastering driving skills and driving automotive technology performance knowledge, the driver must to master the knowledge of road conditions and safe driving for mountainous expressway. The transport enterprises must to enhance driver learning oversight, because drivers generally with low-levels of education and poor active learning. At the same time, the transportation enterprise needs to enrich the driver safe driving technology study content and the form, such as organizing knowledge contests, and driving skills experience symposium. The driver can raise awareness of road safety and to learn knowledge of safe driving and driving an emergency operation techniques, which helps to control traffic accidents from the source.

\section{References}

[1] Bing Chen. Traffic Engineering Technology [M].Chengdu: Southwest Jiaotong University Press.

[2] Yulong Pei, Wei Wang. Road Traffic Accident Causes and Prevention Countermeasures. Beijing: Economic Science Press,2004

[3] JTGB01-2003,Highway Technical Standard[S]. 
[4] [9] Shibo Zhang, Yong Luo, Bin Chen and Xiuchao Bao.Progress of simulation studies of pedestrian traffic. ICTE2009.103-108

[5] Road Safty Manual. Recommendations from the World Road Association(PIARC).2005,P107

[6]Chen bin and Luo yong.The road safety prediction model based on the fuzzy linear regression. CINC2009,142-148 